\title{
Derivati dei nomi dei mesi (Aprile)
}

\author{
Lucia Francalanci
}

PUBBLICATO: 25 APRILE 2021

\section{Quesito:}

Continuiamo la pubblicazione della rubrica dedicata ai derivati dei nomi dei mesi con la scheda relativa ad aprile; chi volesse leggere le schede riguardante i mesi di gennaio, febbraio e marzo può leggere qui, qui e qui. Per le informazioni generali e la bibliografia si rimanda all'Introduzione.

\section{Derivati dei nomi dei mesi (Aprile)}

I 1 nome aprile deriva dall'aggettivo latino aprīlis, la cui etimologia è incerta. L'Etimologico riporta le due proposte esposte nell'Ernout-Meillet (DELL): secondo Cuny, dato che nel calendario romano la metà dei mesi aveva nomi su base numerica e marzo era il primo mese dell'anno, aprîlis potrebbe significare 'il secondo mese', sulla base di un ordinale *aporo- 'secondo, successivo', che ha sicure corrispondenze indoeuropee (sanscrito Aparos, avestico Aparō 'altro, secondo', gotico Afar 'dopo'); secondo Benveniste, aprīlis si inserirebbe nella sequenza dei mesi dedicati alle divinità e sarebbe il mese dedicato a Venere, il cui nome si ricostruisce partendo dal greco Aphrós, ipocoristico di Aphroditē, passato per un intermediario etrusco *Apru; si tratterebbe dunque di un nome etrusco passato nel latino in epoca arcaica.

Come visto per gennaio e febbraio, anche la famiglia di derivati di aprile è particolarmente povera (ma ciò vale pure, come vedremo, novembre e dicembre), con appena sette termini rintracciati, di cui soltanto due attestati dalla lessicografia.

- aprilano

L'aggettivo aprilano non è registrato dai dizionari ma risulta ben attestato in rete e nei testi a stampa; per lo più è usato in riferimento a condizioni meteorologiche (situazione aprilana, giornata aprilana, nevicate aprilane):

Tuttavia non è neppur necessario avere una straordinaria memoria per ricordarsi di altre nevicate marzeline, anzi aprilane, come il 2r aprile 1908 , il 29 aprile 1907 (nevischio), il 3 aprile 1906 , nel quale anno nevicò anche il 21,22, 26 marzo, salendo, il 22, a due centimetri. (Nevicate e temporali, "Corriere della Sera", I/4/r9io)

Nell'esempio precedente compare anche l'aggettivo marzeline, femminile plurale; però un maschile singolare marzelino non sembra essere diffuso né in rete né nei testi a stampa; è probabile che si tratti di un refuso per marzoline.

- aprilante

L'aggettivo aprilante 'del mese di aprile' (derivato diaprile con il suffisso-ante, costruito probabilmente per ragioni di rima) è usato (spesso sostantivato) esclusivamente nel proverbio (e varianti) Quarto (o terzo) aprilante, quaranta di durante, che significa: il tempo che fa il quarto giorno di aprile (o il terzo, nella variante) dura quaranta giorni, cioè serve come pronostico per il periodo 
successivo.

La credenza è diffusa in Europa con una certa regolarità, cosa che fa pensare a origini religiose del detto:

Il numero 40 poi è sempre stato legato alla pioggia a cominciare dalla narrazione del Diluvio: «Piovve sopra la terra per quaranta dì e quaranta notti» (Genesi VII, I2). Il quaranta è sempre stato numero costante di quei periodi necessari all'espiazione e alla penitenza: tanti sono gli anni che gli Ebrei passarono nel deserto, i giorni del digiuno di Cristo, quindi i giorni della quaresima, i giorni tra la Resurrezione e l'Ascensione, quelli da Natale alla Candelora e quelli della quarantena imposta anticamente per le malattie infettive. (Antoni e Lapucci I985, p. I03-I04)

Tra le varianti citiamo anche quelle riportate dai Proverbi toscani di Giuseppe Giusti (terzo d'Aprilante, quaranta di durante) e dai Proverbi di Francesco Serdonati (Trenta di ha Aprilante, quaranta di (di) sembiante), quella presente nel Dizionario dei proverbi italiani di Schwamenthal e Straniero del I99I (Aprile, aprilante, quaranta giorni durante) e le sentenze registrate in Boggione e Massobrio (2004) e nell'Atlante Paremiologico Italiano a cura di Franceschi (Quando piove il due aprilante, si riempiono i pieni e i vuoti; Quando piove i tre aprilanti, piove tutti quanti; Quattro aprilanti, quaranta di duranti).

- aprilata/aprilato

L'aggettivo aprilato, non accolto dai dizionari, risulta poco diffuso anche in rete e nei testi a stampa; una delle poche occorrenze del termine si ha nella traduzione italiana del Dizionario classico di storia naturale di Jean-Baptiste-Georges-Marie Bory de Saint-Vincent (vol. VIII, Venezia, Girolamo Tasso Editore, I836), in cui si fa riferimento al "grano aprilato, quello seminato in aprile" (p. 555). Risulta invece leggermente più diffuso il sostantivo femminile aprilata che, modellato su maggiolata e ottobrata, indica una festa o una scampagnata:

A Roma si parla del bel tempo delle "ottobrate" ma non mai di "aprilate", e la primavera, in quasi tutta l'Italia, divide con l'autunno la caratteristica, utile per l'agricoltura ma molto noiosa per i cittadini, di essere la stagione avente le più alte precipitazioni. (G. Roncali, Noioso ma non eccezionale il maltempo di primavera, "Corriere della Sera", 24/4/1959; l'esempio, peraltro, documenta l'inesistenza della parola)o

- aprilatico/aprilese

Come abbiamo visto per gennaiese/gennarese e per febbraiese/febbrarese, è possibile rintracciare in rete alcuni aggettivi (anche in forma sostantivata), non registrati dai dizionari, usati in riferimento ad alcune colture, che derivano dal mese della raccolta. Ad esempio, il broccolo, il broccoletto, la cima di rapa, il cipolletto e il cavolfiore tipico della zona napoletana includono tra le specie l'aprilatico; la cipolla bianca comprende le tipologie febbrarese, aprilatica e giugnese, ma esiste anche la cipolla aprilese; tra i tipi di trifoglio incarnato troviamo il marzotico, l'aprilatico e il giugnese:

Originaria del bacino del Mediterraneo, questa specie [trifoglio incarnato, ndr] è molto diffusa negli ambienti meridionali ed è particolarmente resistente al freddo. La sua variabilità genetica è elevata, di conseguenza sono noti molti ecotipi classificati con appellativi del periodo di utilizzazione: marzotico, aprilatico, maggenzo [sic], giugnese e lugliese. (dal sito La Buona Terra, Associazione Lombarda Agricoltori Biologici e Biodinamici)

- aprilesco

L'aggettivo aprilesco 'proprio del mese di aprile' non è accolto dai dizionari ma risulta attestato in rete 
e in testi a stampa. Ne riportiamo qualche esempio:

Il barcone colle pompe e motori elettrici, resterà poi ad impianto finito, con scopo identico di quello che vedemmo nell'impianto del Löntsch, e cioè utilizzare ancora più in annate di piogge aprilesche tardive, le acque contenute nella profondità del lago. (Atti della associazione elettrotecnica italiana, Stucchi Ceretti \& C., I9II, p. 706)

C'eravamo alzati ch'era ancora buio, appena spiovuto nella notte aprilesca, e con un corteggio di carrozze s'era imboccata ad andatura regolare, per non destare sospetti, la via per Porta Portese. (Luca Desiato, Il marchese del Grillo, Roma, Newton Compton, 20II, p. 62; prima edizione Milano, Mondadori, 198I)

- aprilino

L'aggettivo aprilino 'proprio, caratteristico del mese di aprile' è marcato dai dizionari sincronici come termine di basso uso, non comune o talvolta come voce letteraria. Il Sabatini-Coletti registra anche l'accezione 'primaverile' e il dizionario Hoepli 2018 l'uso in senso figurato per 'giovane, fresco'. Nel GDLI è riportato un esempio di Ugo Ojetti:

La primavera quest'anno è giunta tardi e le foglie hanno in pieno maggio una trasparenza e una tenerezza apriline. (Ugo Ojetti, Cose viste, opera in 7 voll., articoli scritti per il "Corriere della Sera" dal I923 al 1939)

Nota bibliografica:

- A. Ernout, A. Meillet, Dictionnaire Etymologique de la Langue Latine (DELL), Paris, Klincksieck, 1932

- A. Cuny, LAT. APRĪLIS, "Mémoires de la Société de Linguistique de Paris" (MSL), I4, I9o6I908, pp. 286-288

- E. Benveniste, Trois étymologies latines, "Bulletin de la Société de Linguistique de Paris" (BSL), 32, I93I, pp. 68-73

\section{Cita come:}

Lucia Francalanci, Derivati dei nomi dei mesi (Aprile), "Italiano digitale", XVII, 2021/2 (aprile-giugno)

DOI: $10.35948 / 2532-9006 / 2021.7521$

Copyright 2021 Accademia della Crusca

Pubblicato con licenza creative commons CC BY-NC-ND 